\title{
Application of Alternate Iterative Algorithm to Image Restoration
}

\author{
Xu-Chao LI ${ }^{1, a}$, Song-Yan MA ${ }^{1}$ and Wen-Juan $\mathrm{LI}^{2}$ \\ ${ }^{1}$ College of Computer and Information Engineering, Chifeng University, Chifeng, Inner Mongolia, 024000, China \\ ${ }^{2}$ College of Mathematics and Statistics, Chifeng University, Chifeng, Inner Mongolia, 024000, China
}

\begin{abstract}
For overcoming the disadvantage of total variation for regularization, which easily produces stair effect, an improved weight energy functional regularization model and alternate algorithm is proposed. Because of non-smooth property of total variation semi-norm regularization model, it is impossible to solve primal model directly. For solving the problem, taking advantage of Fenchel transform, the primal model is converted into dual model, resorting to variable splitting technology, the alternating iterative algorithm is deduced. Moreover, the step length updating principle of primal-dual variable is given. Finally, using Camera image blurred by Gaussian noise, Telescope image blurred by system and Poisson noise for image restoration, simulations show the proposed can achieve smaller relative error, deviation error, higher peak of signal-to-noise ratio, and better visual effect.
\end{abstract}

\section{Introduction}

In remote sensing, microscopic imaging, computed tomography imaging etc. The imaging processing can be described by first kind of Fredholm integral equation[1], which is written as

$$
f(X)=\int_{R^{2}} a(X, Y) u(Y) d Y
$$

Where $f(X)$ and $u(Y)$ represent sample signal and ideal signal respectively, $a(X, Y)$ represents point spread function (PSF). Discretizing formula (1), we get the following model

$$
f=A u+\varepsilon
$$

Where $\varepsilon$ is noise, $A$ is linear tight operator with a large condition number, and it is impossible to get effective solution of (2). Moreover, transformation error of imaging device, atmospheric turbulence and random impulse,etc, all of them can make image blurry. For obtaining high quality and meaning image, at present, it is widely accepted to establish reasonable energy functional regularization model (EFRM)[2], which can be described as

$$
E(u)=E_{0}(u)+\alpha \Phi_{0}(B u)
$$

\footnotetext{
${ }^{\text {a }}$ Corresponding author: bsx7096@sohu.com
} 
Where $E_{0}(u)$ is fitting term, $\Phi_{0}(B u)$ is regularization term, $B$ is first or second order differential operator, and $\alpha$ is regularization parameter.

For fitting term, the model is established according to noise statistical distribution of imaging processing[3]. Such as, if $\varepsilon$ is Gaussian noise, fitting term is described by $\mathrm{L}_{2}$ norm, if $\varepsilon$ obeys Poisson distribution, fitting term is measured by Kullback-Leiber function, if $\varepsilon$ is salt and pepper noise, fitting term is approximated by $\mathrm{L}_{1}$ norm[4]. For regularization term, in academic field, it is widely accepted that the model is described by total variation semi-norm, which can accurately describe singular and jump property of solution. Taking advantage of the property, Rudin et al establish ROF model for image restoration[5], which can effectively protect image edges, however, which easily produces stair effect in steady area, moreover, because of the non-smooth property of total variation semi-norm, it is difficult to design effective algorithm for primal EFRM.

For the shortcomings of total variation semi-norm, scholars mainly carry out three aspect researches: 1) Converting the primal EFRM into smooth EFRM. For example, using smooth square root function approximating total variation semi-norm, taking advantage of EFRM differential property, many classic algorithms can be used. Such as the steepest descent iterative algorithm, fixed point iterative algorithm, Newton and quasi-Newton iterative algorithm[6], etc. However, if the gradient magnitude of EFRM is too small, the convergence speed of algorithm is very slow. Moreover, generally speaking, the descriptive question of EFRM is always large scale, and it is difficult to compute the inverse Hessian matrix for Newton iterative algorithm. For overcoming these shortcomings, a lot of scholars divide the integrated EFRM into several sub-problems. For example, differential fitting term forms predictive sub-problem, non-differential regularization term forms proximal sub-problem, two sub-problem alternate iterations form iterative soft threshold algorithm(ISTA)[7], which doesn't need to compute the inverse Hessian matrix, but the convergence speed of algorithm is slower. To accelerate the convergence speed of algorithm, resorting to ISTA idea, reference [8] proposed a fast ISTA (FISTA). Although the convergence speed of FISTA is faster than that of ISTA, the convergence speed of FISTA depends on the characteristic value of $A^{\mathrm{T}} A .2$ ) Using Fenchel transform, converting the primal EFRM into dual EFRM. Based on the idea, reference [9] proposed half implicit gradient descent algorithm, which initiated the application landmark of dual EFRM to image restoration. Simulation shows that the quality of image restoration of dual EFRM is better than that of primal EFRM[10]. 3) Converting the primal EFRM into primal-dual EFRM. Such as primal-dual iterative algorithm[11] with constant, adaptive, and variable step length updating[12]. The image restoration quality of primal-dual EFRM is better than that of dual EFRM. However, if without good initialization, the primal-dual EFRM easily makes the image edge blurry.

This paper is structured as follows: In Section 2, in order to protect image edges, we establish an EFRM with adaptive edge detection weight function. Then, the EFRM is converted into dual EFRM, using variable splitting technology, dividing dual EFRM into primal and dual sub-problems, then, an alternate iterative algorithm with adaptive step length updating scheme is proposed. In Section 3, we focus on the implementation of the proposed algorithm, using blurred image by Gaussian, system and Poisson noise for quantitative and qualitative numerical experiments, and comparing with previous state-of-the-art algorithms. Conclusion about the proposed algorithm is drawn in Section 4.

\section{Establishment EFRM for image restoration}

\subsection{Primal EFRM}

Suppose that the fitting term of (3) is second order differential convex function with Lipschitz continuous condition[13]. For accurately describing the singular and jump characteristics of image, an improved regularization term with adaptive edge detection weight function can be rewritten as

$$
\Phi(B u)=\alpha \int_{\Omega} g(x)|B u| d x
$$


Where $\int_{\Omega}|B u| d x$ is total variation function semi-norm, and it is defined as

$$
\int_{\Omega}|B u| d x=\sup \left\{\int_{\Omega} u \operatorname{div} \phi d x\right\}
$$

Where $x \in \Omega, \phi \in C_{0}^{1}\left(\Omega, R^{2}\right)$ and $|\phi(x)|<1$. div is divergent, $g(x)$ is adaptive weight function, which is defined as

$$
g(x)=\frac{1}{1+K\left|\nabla G_{\sigma} * u\right|^{2}}
$$

Where $K \geq 0, G_{\sigma}=\frac{1}{2 \pi \sigma^{2}} \exp \left(-\frac{x^{2}}{2 \sigma^{2}}\right), \nabla$ is gradient and $*$ is convolution. In steady area of image, the magnitude of $\left|\nabla G_{\sigma} * u\right|$ is bigger, the diffusion coefficient of $g(x)$ is smaller, which is advantageous to image edge. On the contrary, the magnitude of $\left|\nabla G_{\sigma} * u\right|$ is smaller, the diffusion coefficient of $g(x)$ is bigger, which can prevent from producing stair effect in unsteady area. So $g(x)$ can adaptively embody image property. Substituing (4) into (3), we establish an improved primal EFRM, which is formulated as

$$
E(u)=E_{0}(u)+\alpha \Phi(B u)
$$

Expression (7) about $u$ is coercive, according to direct variation principle, the solution of (7) exists. Becaus fitting term and regularization term about $u$ are both convex, the solution of (7) is unique.

\subsection{Converting primal EFRM into primal-dual EFRM}

Expression (7) is non-differential, it is difficult to solve directly. For solving the problem, taking advantage of Fenchel transform, primal EFRM is converted into dual EFRM. Suppose $\Omega \subset H, H$ is Hilbert space, the map $\varphi . X \rightarrow Y$ is proper, convex function, $Y=R \cup\{\infty\}$, Fenchel transform of $\varphi(x)$ is defined as

$$
\varphi^{*}(\xi)=\sup _{\omega \in X}\{\langle\xi, \omega\rangle-\varphi(\omega)\}
$$

Where $\langle\cdot, \cdot\rangle$ is inner product, $\varphi^{*}: \xi \rightarrow R \cup\{\infty\}$ is proper function, if $\varphi^{*}(\xi)$ is lower semicontinuous, then $\varphi^{* *}(\xi)=\varphi$.

$\Phi(B u)$ of (7) is proper, convex, semicontinuous convex function, let $z=B u$, then

$$
\Phi(z)=\alpha \int_{\Omega} g(x)|z| d x
$$

Suppose the dual variable of $z$ is $z^{*}$, according to (8), the dual expression of (9) is expressed as

$$
\Phi^{*}\left(z^{*}\right)=\max _{z}\left\{\left\langle z^{*}, z\right\rangle-\Phi(z)\right\}=\max _{z}\left\{\left\langle z^{*}, z\right\rangle-\alpha \int_{\Omega} g(x) z \mid d x\right\}=\left\{\begin{array}{cc}
0 & \text { if }\left|z^{*}\right| \leq \alpha g(x) \\
+\infty & \text { otherwise }
\end{array}\right.
$$

Because $\Phi^{*}\left(z^{*}\right)$ is proper, lower semicontinuous convex function, $\Phi^{* *}(z)$ is the conjugation of conjugation of $\Phi(z)$, according to (8), then $\Phi^{* *}(z)=\Phi(z)$. Let $q=z^{*}$, according to (10), then 


$$
\Phi(z)=\max _{q}\left\{\langle q, z\rangle-\Phi^{*}(z)\right\}
$$

$z$ Replaced by $B u$ in (11), substituing (11) into (7), the primal-dual EFRM is expressed as

$$
\min _{u} \max _{q}\left\{E_{0}(u)+\alpha\lfloor\langle q, B u\rangle\rfloor-\Phi^{*}(q)\right\}
$$

Where $q=\left\{\left(q_{1}, q_{2}\right)^{T}|| q(x) \leq \alpha g(x)\right\}$.

\subsection{Alternate iterative algorithm for primal-dual EFRM}

Expression (12) is Min-Maximum problem, by performing the maximization over $q$ in (12), and we get the dual variable sub-problem

$$
\max _{q}\left\{\alpha\lfloor\langle q, B u\rangle\rfloor-\Phi^{*}(q)\right\}
$$

Similary, performing the minimization over $u$ in (12), we get the primal variable sub-problem

$$
\min _{u}\left\{E_{0}(u)+\alpha\lfloor\langle q, B u\rangle]\right\}
$$

The iterative algorithm is described as

Initialization: $\delta_{0}, \tau_{0}, u_{0}, q_{0}, k$, maximum iterative number $n u m=1000$.

Loop iteration:

1)The dual sequences $\left\{q_{k}\right\}$ is updated as follows

$$
q_{k+1}=\left(1+\delta_{k} \alpha \partial \Phi^{*}\right)^{-1}\left(q_{k}+\delta_{k} \alpha B \overline{u_{k}}\right)
$$

Where $\delta$ is step length of dual variable, $\left(1+\delta_{k} \alpha \partial \Phi^{*}\right)^{-1}$ is proximal operator, $\partial \Phi^{*}$ is sub-gradient.

2)The primal sequences $\left\{u_{k}\right\}$ is updated as follows

$$
u_{k+1}=P_{X}\left(u_{k}-\tau_{k} B^{T} q_{k}\right)
$$

Where $\tau$ is step length of primal variable, $P_{X}(r)$ is projection operator, which is defined as

$$
P_{X}(r)=\underset{x \in X}{\operatorname{argmin}} \frac{1}{2}\|x-r\|^{2}
$$

3)The auxiliary sequences $\left\{\bar{u}_{k}\right\}$ is updated as follows

$$
\bar{u}_{k+1}=\bar{u}_{k}+\theta\left(u_{k+1}-\bar{u}_{k}\right)
$$

Where $\theta \in[0,1]$.

Termination conditions:

If $k>$ num or $\left\|E\left(u_{k+1}, q_{k+1}\right)-E\left(u_{k}, q_{k}\right)\right\| \leq t$, output $u_{k}$. Otherwise, $k=k+1$, updating $\delta_{k}, \tau_{k}$. 


\subsection{Step length updating principle}

According to (15) and (16), Step length updating principle produces profound influence on alternate iterative algorithm convergence. Supposed the fitting term satisfies Lipschitz continuous condition, step length series of $\left\{\delta_{k}\right\},\left\{\tau_{k}\right\}$ must satisfy the following conditions

$$
\begin{aligned}
& \lim _{k \rightarrow \infty} \tau_{k}=0, \sum_{k=0}^{\infty} \tau_{k}=\infty, \lim _{k \rightarrow \infty} \delta_{k}=\infty \\
& \sum_{k=0}^{\infty} \frac{\tau_{k}}{\sigma_{k}}<+\infty, \sum_{k=0}^{\infty} \tau_{k}^{2}<+\infty
\end{aligned}
$$

If alternate iterative algorithm satisfies (19) and (20), the iterative series $\left(u_{k}, q_{k}\right)$ of (15)-(18) will converge to the optimum solution $\left(u^{*}, q^{*}\right)$ of (12).

\section{Experiment results and analysis}

In order to measure image restoration quality, three kinds of performance index, mean absolute error (MAE), peak signal-to-noise ratio (PSNR) and relative error (RE), are respectively defined as

$$
\begin{aligned}
& \text { MAE }=\frac{1}{m n} \sum_{i=1}^{m} \sum_{j=1}^{n}\left|u_{i j}^{(k)}-u_{i j}\right| \\
& \text { PSNR }=10 \log _{10}\left(\|\left.\max \left(u_{i j}\right)\right|_{2} ^{2}\right) / m n \| u_{i j}^{(k)}-\left.u_{i j}\right|_{2} ^{2} \\
& \mathrm{RE}=\left\|u_{i j}^{(k)}-\left.u_{i j}\right|_{2} ^{2} /\right\| u_{i j} \|_{2}^{2}
\end{aligned}
$$

Where $u$ is original image, $u_{k}$ is restoration image, $m \times n$ is image size. Generally, if PSNR is larger, MAE and RE is smaller, the restoration quality of image is better. On the contrary, the quality is worse.

Experiment 1: For image blurred by Gaussian noise, set $A=I$, the fitting term of (12) is described by $E_{0}(u)=\|u-g\|_{2}$, according to (17)-(18), the step length sequences of primal and dual are updated as follows

$$
\tau_{k}=\frac{\eta_{k}}{1-\eta_{k}}, \delta_{k}=0.2+0.08 k
$$

Where $\eta_{k}=\left(0.5-\frac{5}{15+k}\right) / \delta_{k}$.

The image is Camera of size $256 \times 256$, and the grey level is $0-255$. The blurred version of Camera is shown in Figure 1(b) with PSNR=22.081db. To assess the relative merit of the proposed, we compare the restored results by the proposed strategy with those of classical Landweber iterative algorithm[6], ROF model[5], Chambolle dual model[9].

The qualitative results are shown in Figure 1. In Figure 1(c), the restored image of Landweber algorithm is more smoother than original image, and makes a lot of edges lost. Figure 1(d), the restored image of ROF model produces a lot of staircasing artificts in smooth regions. Figure 1(e), using Chambolle dual model for restoration image, the visual effect is improved, but in smooth regions, a lot of pseudo-trances are produced. Figure 1(f), using the proposed for restoration image, the visual effect is better than that of previous three algorithms. 


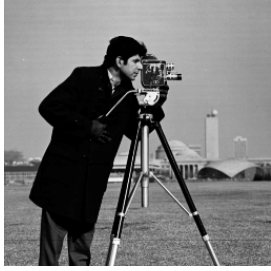

(a) original image

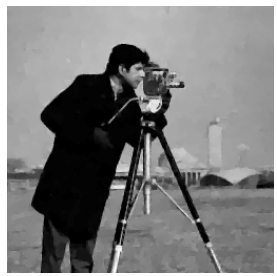

(d) ROF model

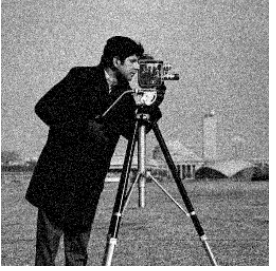

(b) blurry image

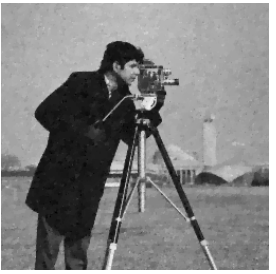

(e) Chambolle dual model

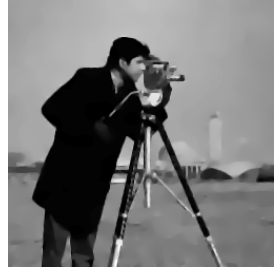

(c) Landweber algorithm

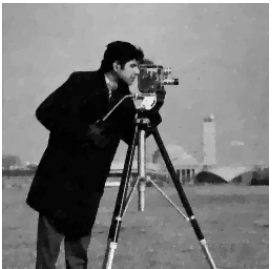

(f) the proposed algorithm

Figure 1. Restoration comparison of different algorithms

According to the quantitative performance index of Table 1, Landweber algorithm obtains the lowest PSNR, the highest RE and MAE, which shows that the quality of image restoration is worse. PSNR of Chambolle dual model is higher than that of ROF model, RE is lower than that of ROF model, but MAE is higher than that of ROF model. The proposed obtains the highest PSNR, the smallest RE and MAE, which show that the quality of image restoration is best.

Table 1. comparison of different restoration algorithms for blurred image by Gaussian noise

\begin{tabular}{ccccccc}
\hline $\begin{array}{c}\text { original } \\
\text { image }\end{array}$ & index & $\begin{array}{c}\text { Blurry } \\
\text { Fig.1(b) }\end{array}$ & $\begin{array}{c}\text { Landweber } \\
\text { Fig.1(c) }\end{array}$ & $\begin{array}{c}\text { ROF model } \\
\text { Fig.1(d) }\end{array}$ & $\begin{array}{c}\text { Chambolle } \\
\text { Fig.1(e) }\end{array}$ & $\begin{array}{c}\text { The propose } \\
\text { Fig.1(f) }\end{array}$ \\
\hline \multirow{2}{*}{ Fig.1 } & RE & 0.1485 & 0.0918 & 0.0754 & 0.0701 & 0.0657 \\
& MAE & 15.909 & 6.6955 & 5.8901 & 5.9578 & 5.4845 \\
& PSNR & 22.081 & 26.258 & 27.972 & 28.595 & 2.9168 \\
\hline
\end{tabular}

Experiment 2: For image blurred by Poisson noise, the fitting term of (12) is described by $E_{0}(u)=\|A u-g \log A u\|_{1}$, according to (19)-(20), the step length sequences of primal and dual are updated as follows

$$
\tau_{k}=0.5 / \delta_{k}, \quad \delta_{k}=0.2+0.08 k
$$

The image is Telescope of size $256 \times 256$, and the grey level is $0-255$. According to the Poisson property, the PSF of size $128 \times 128$ and $256 \times 256$ are constructed respectively. We compare the restoration results of the proposed algorithm with quasi-Newton algorithm.

Taking advantage of psfGaussian function[14] for producing PSF of size $128 \times 128$ and $256 \times 256$, they are shown in Figure 2(a) and 2(b), respectively. Figure 3 is singular value decomposition (SVD) of PSF, circle and solid line represent matrix SVD of Figure 2(a) and Figure 2(b), respectively. Figure 3 shows the condition number of marix $A$ is ill-posed, which satisfies the condition of (1) and (2). 


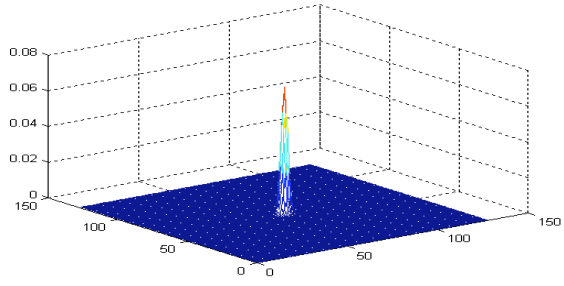

(a) PSF of size $128 \times 128$

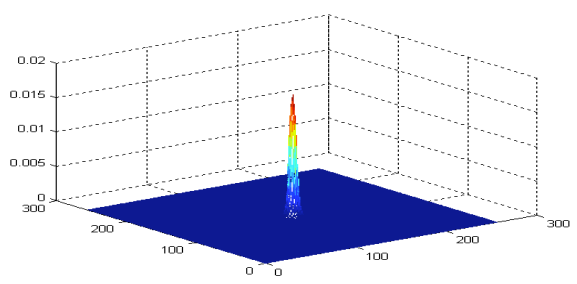

(b)PSF of size $256 \times 256$

Figure 2. PSF with different size

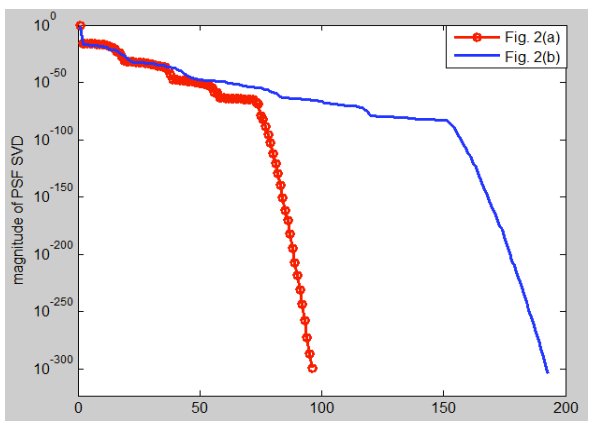

Figure 3. SVD of PSF

Figure 4(a) is original telescope image, Figure 4(b) is blurred by Figure 2(a) of PSF and Poisson noise. According to qualitative results, reference [10] algorithm produces a lot of pseudo-trances, as shown in Figure 4(c). in Figure 4(d), the visual effect of the proposed is better than that of reference [10].

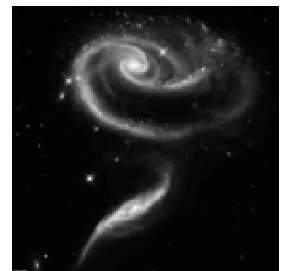

(a) original image

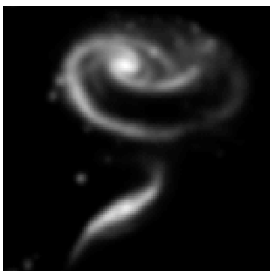

(b) blurry image

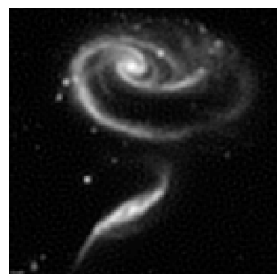

(c) reference [10] algorithm

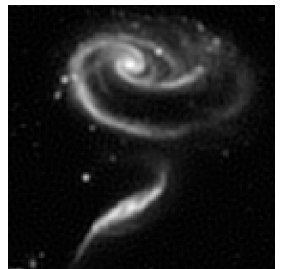

(d) the proposed

Figure 4. Restoration comparison of different algorithms $(128 \times 128)$

According to the quantitative performance index of table 2, PSNR of the proposed is higher than that of reference [10] algorithm, and RE and MAE of the proposed are lower than that of reference [10] algorithm. The quantitative results shows that image restoration quality of the proposed is better than that of reference [10] algorithm.

Table 2. comparison of restoration image blurred by Poisson noise

\begin{tabular}{ccccc}
\hline $\begin{array}{c}\text { Original } \\
\text { image }\end{array}$ & index & $\begin{array}{c}\text { Blurry image } \\
\text { Figure 4(b) }\end{array}$ & $\begin{array}{c}\text { Reference [10] } \\
\text { Figure 4(c) }\end{array}$ & $\begin{array}{c}\text { The proposed } \\
\text { Figure 4(d) }\end{array}$ \\
\hline 4(a) & RE & 0.1228 & 0.0755 & 0.0691 \\
Telescope & MAE & 0.0113 & 0.0116 & 0.0098 \\
$128 \times 128$ & PSNR & 31.443 & 35.668 & 36.433 \\
\hline
\end{tabular}


Figure 5(a) is original telescope image, Figure 5(b) is blurred by Figure 2(b) of PSF and Poisson noise, it is blurred more serious than figure 4(b). According to qualitative results, reference [10] algorithm produces lots of pseudo-trances in Figure 5(c), it is more serious than Figure 4(c). Visual effect of restoration of Figure 5(d) is better than that of Figure 5(c).

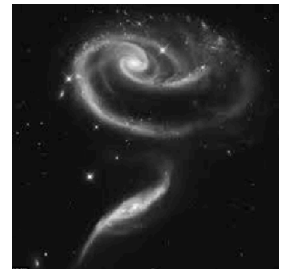

(a) original image

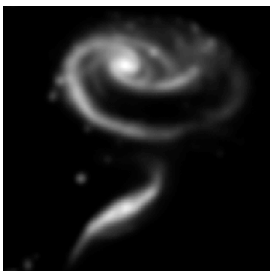

(b) blurry image

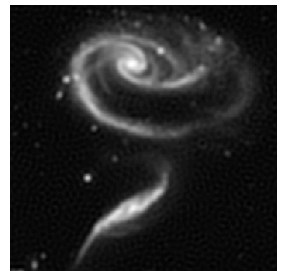

(c) reference [10] algorithm

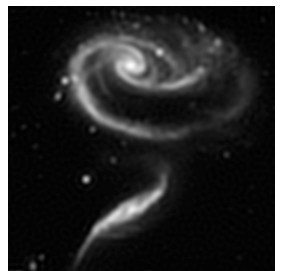

(d) the proposed

Figure 5. Restoration comparison of different algorithms $(256 \times 256)$

According to the quantitative performance index of table 3, PSNR of the proposed is higher than that of reference [10] algorithm, and it is improved about $0.3 \mathrm{db}$. RE and MAE of the proposed are lower than that of reference [10] algorithm.

Table 3. comparison of restoration image blurred by Poisson noise

\begin{tabular}{ccccc}
\hline $\begin{array}{c}\text { Original } \\
\text { image }\end{array}$ & index & $\begin{array}{c}\text { Blurry image } \\
\text { Figure 5(b) }\end{array}$ & $\begin{array}{c}\text { Reference [10] } \\
\text { Figure 5(c) }\end{array}$ & $\begin{array}{c}\text { The proposed } \\
\text { Figure 5(d) }\end{array}$ \\
\hline $5(\mathrm{a})$ & RE & 0.1370 & 0.0849 & 0.0825 \\
Telescope & MAE & 0.0123 & 0.0109 & 0.0090 \\
$256 \times 256$ & PSNR & 30.760 & 34.917 & 35.164 \\
\hline
\end{tabular}

\section{Conclusion}

The paper proposes an improved weight EFRM, taking advantage of Fenchel transform, converting the primal model into dual model, resorting to splitting technology, and deducing an alternate iterative algorithm. Experiment results show the algorithm can effectively restore image blurred by Gaussian noise, system and Poisson noise, and obtain smaller RE and MAE, the larger PSNR.

\section{Acknowledgement}

This work was supported by Higher Educational Scientific Research Projects of Inner Mongolia Autonomous Region, China (NJZY16254), and project supported by the Natural Science Foundation of Inner Mongolia Autonomous Region, China (2016MS0602).

\section{References}

1. Bai zhengjian, Donatelli Marco, Capizzano Stefano Serra, Fast preconditioners for total variation deblurring with anti-reflective boundary conditions, SIAM Journal on matrix analysis and applications, 32, 3(2011)

2. Serafini Thomas, Zanghirati Gaetano, Zanni Luca, Gradient projection methods for large quadratic programs and applications in training support vector machines, Optimization Methods and Software, 20, 2-3(2004)

3. Du Z M, Li H A, Kang B S, Ye F Y, Recovery method of compressed sensing signals based on quadratic convergence regularization with a smooth functional[J], Journal of Image and Graphics, 21, 4(2016). 
4. Li X C, Bian S X, Li Y Y, Survey of convex energy functional regularization model of image restoration[J], Journal of Image and Graphics, 21, 4(2016).

5. Leonid I. Rudin, Stanley Osher, Emad Fatemi, Nonlinear total variation based noise removal algorithms, Physical D: Nonlinear Phenomena, 60, 1(1992)

6. Curtis R. Vogel, Computational methods for inverse problems(SIAM: Society for Industrial and Applied Mathematics, 2002)

7. Combettes P. L, V. Wajs, Signal recovery by proximal forward-backward splitting, SIAM Journal on Multiscale Modeling and Simulation, 4, 4(2005)

8. Beck Amir, Teboulle Marc, A fast dual proximal gradient algorithm for convex minimization and applications, Operations Research Letters, 42, 1(2014)

9. Antonin Chambolle, An algorithm for total variation minimization and applications, Journal of Mathematical Imaging and Vision, 20, 1-2(2004)

10. Duran Joan, Coll Bartomeu, Sbert Catalina, Chambolle's projection algorithm for total variation denoising, Image Processing on Line, 3, (2013)

11. Antonin Chambolle, Thomas Pock, A first-order primal-dual algorithm for convex problems with applications to imaging, Journal of Mathematical Imaging and Vision,40,1( 2011)

12. Goldstein Tom, Esser Ernie, Baraniuk Richard, Adaptive primal-dual hybrid gradient methods for saddle- point problems, http://arxiv.org/abs/1305. 0546 (2013)

13. Gilles Aubert, Pierre Kornprobst, Mathematical problems in image processing, partial differential equations and the calculus of variations, second edition (Springer-Verlag, New York, USA, 2006)

14. Katrina Palmer Lee, James G. Nagy, Lisa Perrone, Iterative methods for image deblurring: a matlab object- oriented approach, Numerical Algorithms, 36, 1(2004) 